\title{
Endoscopic treatment of GERD: is there still a chance?
}

\section{다)(우우}

\author{
Authors \\ Pietro Familiari ${ }^{1,2}$, Francesca Mangiola ${ }^{1}$, Rosario Landi ${ }^{1}$, Guido Costamagna ${ }^{1,2}$ \\ Institutions \\ 1 Digestive Endoscopy Unit, Fondazione Policlinico \\ Universitario A. Gemelli IRCCS, Rome, Italy \\ 2 Centre for Endoscopic Research Therapeutics and \\ Training - CERTT, Università Cattolica del Sacro Cuore, \\ Rome, Italy \\ Bibliography \\ DOI https://doi.org/10.1055/a-1012-1905 | \\ (c) Georg Thieme Verlag KG Stuttgart · New York \\ elSSN 2196-9736 \\ Corresponding author \\ Dr. Pietro Familiari, Digestive Endoscopy Unit, Fondazione \\ Policlinico Universitario A. Gemelli IRCCS, Largo Gemelli 8, \\ 00168 Rome, Italy \\ Fax: +390630156581 \\ pietrofamiliari@tiscali.it
}

Endoscopy International Open 2019; 07: E1701-E1703
Gastroesophageal reflux disease (GERD) is the most common esophageal disorder, affecting more than $20 \%$ of the population worldwide. GERD symptoms vary from simple heartburn and epigastric pain to more complex clinical situations, with a combination of other various extra-esophageal symptoms that include hoarseness, odynophagia, chronic cough, pharyngitis and laryngitis, asthma, disrupted sleep, chronic bronchitis and pneumonia.

Current medical and surgical therapies are adequate treatments for the vast majority of patients with GERD. Proton pump inhibitors (PPI), in particular, are very effective for treatment of typical esophageal symptoms, induce healing of esophagitis, and they prevent recurrences. Nevertheless, in some patients, symptoms are refractory to standard medical therapy. Furthermore, some concerns about possible longterm effects of therapy with PPI now are emerging, and some patients refuse the idea of a lifetime commitment to medical therapy. Laparoscopic Nissen fundoplication is the most common antireflux surgical operation. At short- and mid-term, surgery offers excellent control of symptoms. In contrast, surgery remain a moderately invasive treatment for medical disorders, and carries a certain risk of immediate and long-term complications. Furthermore, the antireflux wrap may be insufficient to control symptoms after more than 5 years, and patients may need to resume medical therapy.

At the beginning of the current century, a variety of endoscopic antireflux procedures were ideated, developed and put on the market, to respond to the emerging clinical needs imposed by non-response to PPI therapy of patients with GERD or their reluctance to accept lifelong therapy or surgery.

In truth, despite initial enthusiasm for and very promising results with preliminary clinical trials, many of these procedures were quickly abandoned because of serious security warnings, or manifested inefficacy.

A common inexorable destiny united the procedures that consisted in the injection or deployment of inert bulking agents at the level of the esophagogastric junction (Enterix, Boston Scientific, United States and Gatekeeper, Medtronic, United States) and those that permitted creation of superficial plications of tissue at the level of the esophagogastric junction (EndoCinch - CR Bard Inc, United States and the Endoscopic Suturing Device - ESD, Wilson-Cook Medical, United States).

Only three of the endoscopic antireflux procedures developed almost 20 years ago are still available today: the Strettaprocedure (Restech Corporation, Houston, Texas, United States), the NDO Full-Thickness Plicator (that in truth has been acquired by a new company, re-engineered, and proposed in an, almost completely, new guise - the GERDX'M - G-SURG GmbH, Seeon-Seebruck, Germany), and the Esophyx Transoral Incisionless Fundoplication (EndoGastric Solutions, Redmond, Washington, United States).

The first one has a peculiar mechanism of action, actually in large part not completely clarified, and includes delivery of radiofrequency energy at different levels into the LES, by using a special balloon - basket assembly with four retractable needle electrodes arranged radially around the balloon. The latter two devices permit full-thickness plication, with a serosa-to-serosa apposition of tissue, and thus offer more reliable and durable sutures. In addition to these, in more recent years, two other new procedures have become popular: the MUSEsystem (Medigus Ultrasound Surgical Endostapler, Medigus, Omer, Israel) and the ARMS Procedure (Antireflux Mucosectomy): two new kids-on-the-block to reinforce the endoscopic transoral armamentarium against GERD. 
The Stretta Procedure was developed and launched in 1998. However, the original company had financial problems, and their products were acquired by different other companies, most recently in 2019. Nevertheless, the device has never been recalled, discontinued or pulled off the market because of safety concerns or inefficacy. Stretta is still the endoscopic antireflux procedure that has been used the most (probably more than 25.000 cases worldwide). Only very few adverse events have been reported and the efficacy of the procedure was documented by many papers and review, even at longterm follow-up [1].

However, the mechanism of action of the procedure is not completely known. The delivery of radiofrequency does not cause neurolysis, or substantial scarring, fibrosis or wall thickening. On the other hand, it seems to reduce LES compliance and frequency of transient LES relaxations that should be one of the main causes of GERD in patients without hiatal hernia.

A recent meta-analysis confirmed significant improvement in quality of life of treated patients, in association with an improvement in GERD symptoms, reduction in the esophagitis rate by $24 \%$, and a relevant reduction in pathological esophageal acid exposure. At a mean 25-month follow-up, only $49 \%$ of patients required PPIs compared to baseline [1].

GERD-X is the heir of the NDO-Plicator. With this device, a plication is created between the anterior gastric wall and the fundus, under direct endoscopic control. Exciting results came from the initial published series. However, some serious adverse events in the initial experiences quickly brought down Plicator's popularity.

A few years ago, the product was acquired by a German company, and the device completely reengineered. The current GERD-X is disposable, controlled by precise hydraulic movements, and has a more user-friendly handle [2]. Nevertheless, additional studies are still awaited on this new version of the device.

The Transoral Incisionless Fundoplication (TIF) created with the EsophyX device attempts to more closely mimic the effects of antireflux surgery. Under endoscopic control, several fullthickness polypropylene fasteners are deployed between the gastric fundus and the esophagus. The angle of His is elongated, small hiatal hernia reduced, and rotation and fixation of the gastric fundus on the intra-abdominal part of the esophagus creates a new omega-shaped, gastroesophageal valve, similar to a surgical fundoplication. The valve is usually $3.5 \mathrm{~cm}$ long and $200-270^{\circ}$ in circumference. Anatomical changes induced by the fundoplication seem stable and durable.

In a recent meta-analysis of a large number of patients, the procedure was effective in controlling GERD symptoms, with a substantial improvement of the DeMeester score, and $89 \%$ of patients discontinued PPI after the procedure [3]. The results seem durable: at 10 years follow-up, $91.7 \%$ of patients have at least halved their dose of PPI compared to baseline [4].

When compared to surgery, TIF has the highest probability of increasing quality of ILife of patients, but surgery has the highest chances of reducing pathologic esophageal acid exposure time at $\mathrm{pH}<4$ and increasing LES pressure [5].
Both the GERDX and Esophyx should be performed by two expert operators, the first one handling the suturing machine, the other one the endoscope that is used for the direct endoscopic control.

The MUSE system was developed to facilitate and make more standardized and reliable creation of an endoscopic fundoplication. The MUSE system is a concentrate of technology and combines in a single instrument a single-use forward-viewing video-endoscope, an ultrasound system, and a surgical stapler. The surgical staples are fired between the gastric fundus and the distal esophagus, with the goal of elongating the angle of His, and creating a partial anterior fundoplication. The entire procedure is guided and controlled by a computer to minimize inter-operator variability, avoid mistakes, and make the procedure easy and reliable.

Preliminary results of a multicenter registry study, with 1year follow-up demonstrated normalization of pathologic esophageal acid exposure in $16 \%$ of patients and a substantial reduction in $68 \%$, with $70 \%$ of patients who have at least halved their dose of PPI compared to the preoperative period [6].

The last (family of) endoscopic antireflux procedures were ideated by chance when treating some patients with circumferential Barrett's esophagus with cap-assisted EMR or ESD. Scarring after the vast mucosal resection induced a narrowing of the esophagogastric junction, which significantly limited the episodes of reflux. The procedure was called ARMS - antireflux mucosectomy - and preliminary studies showed very encouraging results [7]. However, a circumferential mucosal resection on the gastric side of the cardia is still technically demanding for many endoscopists. Thus the procedure underwent some modifications: the resection area was reduced and others preferred the multiband ligation-assisted EMR or the Argon Plasma Coagulation for mucosal ablation instead of resection [8]. This procedure seems indicated for patients without hiatal hernia, with a slightly enlarged esophagogastric junction. Studies with long-term follow-up and in a large sample of patients are still awaited, but the concept of this procedure is interesting. The procedure is probably less expensive than other endoscopic alternatives, and with proper training and standardization of treatment, can be extremely quick and reliable.

However, even if many of the current endoscopic antireflux procedures have been on the market for almost two decades, many doubts and uncertainties still limit their widespread use. First, anatomical changes induced by the sutures may be not so durable, and the failure of the sutures may be responsible for early recurrence of symptoms. Second, the mechanism of action of some procedures is not completely clear, which is a hard pill to swallow. Finally, and most important, in the vast majority of published studies, clinical efficacy has been calculated by using very subjective and questionable methods: improvement in quality of life and consumption of antacid medications. Usually, a procedure was considered successful when the patient halved the dose of PPI they had used prior to the intervention.

Especially in countries with a public health-care system, one can argue whether it is right and ethical to submit patients to potentially suboptimal treatments when current medical and 
surgical therapies seem more reliable. Controversial methods of evaluating clinical efficacy of an endoscopic antireflux procedure may appear foolish to enthusiastic supporters of evidencebased medicine and "well-done clinical trials" although these methods focus on real clinical needs. Is it a good or bad result if, after surgery, a PPI-dependent GERD patient still takes antacid medication occasionally, for a limited period of time, maybe during spring and autumn? We think that, if we can improve the quality of life of patients with minimal effort, and without serious risks, we should go down this road.

Furthermore, endoscopic antireflux procedures can be repeated (without major difficulties) in case of partial improvement of symptoms or recurrence. Moreover, endoscopic interventions should not be interpreted as a complete alternative to current medical and surgical therapies. They should be considered more like complementary treatments.

In an ideal flowchart, definitely not validated, treatment with PPI should be preferred in patients with occasional, moderately sever symptoms that respond to therapy. In patients who are PPI-dependent or in partial PPI-responders, if EGD reveals no substantial abnormalities of the esophagogastric junction (ie: hiatal hernia or markedly enlarged EGJ), a STRETTA procedure may be preferable.

In contrast, if EGD reveals an enlarged and insufficient EG] or a small hiatal hernia $(1-3 \mathrm{~cm})$, a transoral incisionless fundoplication with Esophyx, MUSE or GERD-X may be more appropriate. As an alternative, in patients without hiatal hernia, an ARMS procedure could be proposed. Surgery could be reserved for patients with larger hiatal hernia, with regurgitation, or as a last resort in case of failure with transoral fundoplication.

Still uncertain is the treatment of prevalent extra-esophageal symptoms, chronic cough, sore throat, asthma, hoarseness, suspected gastro-laryngeal reflux, non-response to classic therapy with PPI, antacids and medical devices with a barrier effect. Researchers involved in trials of endoscopic antireflux procedures noted a common experience: The vast majority of patients who crowded their clinics and asked for these procedures had extraesophageal and atypical symptoms that did not respond o PPI. Unfortunately, the vast majority of these symptoms are more likely related to functional disorders and hypersensitive esophagus than to GERD. Within this group of patients, identification of good candidates for antireflux procedures is far from being easy, and the esophageal $\mathrm{pH}$-impedance study is not always fully reliable and really helpful.

Primum non nocere. If an operative procedure were to be chosen for these patients, probably the STRETTA procedure would be the most appropriate: at worst, it would not work, and symptoms would remain unchanged. It has no relevant side effects, and does not induce any anatomical change.

In conclusion, even if results of the current endoscopic antireflux procedures are not fully convincing, we prefer to see the glass half full. Notwithstanding the doubts regarding the steadfastness of the anatomical changes induced by the sutures, the mechanism of action of some procedures and the uncertainties of clinical efficacy, the advantages and benefits of these procedures should not be underestimated.

The number of patients with GERD is massive. In many western countries, the national cost for PPI and antacids is second only to the expense for antihypertensive drugs. Surgery is costly, more invasive, and patients usually prefer to avoid it. The space left for endoscopic antireflux interventions is enormous, and despite the big shadows on the results and the many limits of the currently available procedures, we think that we should still go down this path and continue with the clinical research in this field.

\section{Competing interests}

None

\section{References}

[1] Fass R, Cahn F, Scotti D] et al. Systematic review and meta-analysis of controlled and prospective cohort efficacy studies of endoscopic radiofrequency for treatment of gastroesophageal reflux disease. Surg Endosc 2017; 31: 4865-4882

[2] Weitzendorfer M, Spaun GO, Antoniou SA et al. Clinical feasibility of a new full-thickness endoscopic plication device (GERDx) for patients with GERD: results of a prospective trial. Surg Endosc 2018; 32: 2541 2549

[3] McCarty TR, Itidiare M, Njei B et al. Efficacy of transoral incisionless fundoplication for refractory gastroesophageal reflux disease: a systematic review and meta-analysis. Endoscopy 2018; 50: 708-725

[4] Testoni PA, Testoni S, Distefano G et al. Transoral incisionless fundoplication with EsophyX for gastroesophageal reflux disease: clinical efficacy is maintained up to 10 years. Endosc Int Open 2019; 7: E647E654

[5] Richter JE, Kumar A, Lipka S et al. Efficacy of Laparoscopic Nissen Fundoplication vs transoral incisionless fundoplication or proton pump inhibitors in patients with gastroesophageal reflux disease: a systematic review and network meta-analysis. Gastroenterology 2018; 154: 1298-1308 e1297

[6] Lankarani A, Costamagna G, Boskoski I et al. Updated results from an international multi-center registry study for endoscopic anterior fundoplication. Gastrointest Endosc 2018; 87: AB282

[7] Inoue $\mathrm{H}$, Ito $\mathrm{H}$, Ikeda $\mathrm{H}$ et al. Antireflux mucosectomy for gastroesophageal reflux disease in the absence of hiatus hernia: a pilot study. Ann Gastroenterol 2014; 27: 346-351

[8] Hedberg HM, Kuchta K, Ujiki MB. First experience with banded antireflux mucosectomy (ARMS) for GERD: feasibility, safety, and technique (with video). J Gastrointest Surg 2019; 23: 1274-1278 